\title{
Doenças de caprinos diagnosticadas na região Central no Rio Grande do Sul: 114 casos $^{1}$
}

\author{
Fábio B. Rosa², Rafaela A. Caprioli ${ }^{3}$, Taiara M. Silva ${ }^{3}$, Glauco J.N. Galiza ${ }^{2}$, \\ Claudio S.L. Barros ${ }^{4}$, Luiz F. Irigoyen ${ }^{4}$, Rafael A. Fighera ${ }^{4}$ e Glaucia D. Kommers ${ }^{4 *}$
}

\begin{abstract}
Rosa F.B., Caprioli R.A., Silva T.M., Galiza G.J.N., Barros C.S.L., Irigoyen L.F., Fighera R.A. \& Kommers G.D. 2013. [Diseases of goats diagnosed in the Central region of Rio Grande do Sul, Brazil: 114 cases.] Doenças de caprinos diagnosticadas na região Central no Rio Grande do Sul: 114 casos. Pesquisa Veterinária Brasileira 33(2):199-204. Laboratório de Patologia Veterinária, Departamento de Patologia, Universidade Federal de Santa Maria, Camobi, Santa Maria, RS 97105-900, Brazil. E-mail: glaukommers@yahoo.com

A retrospective study of the goat necropsies performed over a period of 48 years (1964 to 2011) at the Laboratório de Patologia Veterinária, Universidade Federal de Santa Maria, Rio Grande do Sul (RS) state, Brazil, was performed. A total of 114 reports of goat necropsies were analyzed. Ninety five necropsies (83.33\%) had a conclusive diagnosis and 19 $(19.66 \%)$ had inconclusive diagnosis. Out of the conclusive cases, infectious and parasitary diseases were the most prevalent, followed, in decreasing order of prevalence, by metabolic and nutritional diseases, poisonings and toxi-infections, and developmental diseases. Other conditions or lesions that did not fit any of the above groups of diseases affected about $10 \%$ of the necropsied goats. Hemonchosis was the most prevalent cause of death in this study. Eimeriosis and listeriosis were also important causes of goat deaths. Among the metabolic and nutritional diseases, urolithiasis, osteoporosis, pregnancy toxemia, malnutrition, and white muscle disease were the most prevalent. Mostly the infectious and parasitary diseases and the metabolic and nutritional diseases occurred many times as outbreaks, causing even more important economic losses.
\end{abstract}

INDEX TERMS: Diseases of goats, veterinary pathology.

RESUMO.- Foi realizado um estudo retrospectivo dos arquivos do LPV-UFSM e examinados os laudos de necropsias de caprinos realizadas num período de 48 anos (1964 a 2011). Foram analisados 114 laudos de necropsias de caprinos. Deste total, $95(83,33 \%)$ tinham diagnóstico conclusivo e $19(19,66 \%)$ tinham diagnóstico inconclusivo. Dos 95 casos conclusivos, as doenças infecciosas e parasitárias foram as mais prevalentes, seguidas em ordem decrescente de

\footnotetext{
${ }^{1}$ Recebido em 18 de dezembro de 2012.

Aceito para publicação em 24 de janeiro de 2013.

Parte da Dissertação de Mestrado do primeiro autor.

${ }^{2}$ Programa de Pós-Graduação em Medicina Veterinária, área de concentração em Patologia Veterinária, Centro de Ciências Rurais (CCR), Universidade Federal de Santa Maria (UFSM), Camobi, Santa Maria, RS 97105900, Brasil.

${ }^{3}$ Curso de Medicina Veterinária, CCR-UFSM, Santa Maria, RS.

${ }^{4}$ Laboratório de Patologia Veterinária, Departamento de Patologia, Centro de Ciências da Saúde, UFSM, Av. Roraima 1000, Santa Maria, RS 97105900. *Autor para correspondência: glaukommers@yahoo.com
}

prevalência, pelas doenças metabólicas e nutricionais, intoxicações e toxi-infecções e alterações do desenvolvimento. Outras alterações de diferentes naturezas e etiologias que não se enquadravam nos grupos de doenças acima afetaram cerca de $10 \%$ dos caprinos examinados. A hemoncose foi a principal causa de morte de caprinos na área de abrangência do LPV-UFSM. Eimeriose e listeriose também foram causas importantes de morte. Dentre as doenças metabólicas e nutricionais, urolitíase, osteoporose, toxemia da prenhez, desnutrição e doença dos músculos brancos foram as mais prevalentes. Principalmente as doenças infecciosas e parasitárias e as metabólicas e nutricionais ocorreram muitas vezes na forma de surtos, acarretando maiores perdas econômicas associadas.

TERMOS DE INDEXAÇÃO: Doenças de caprinos, patologia veterinária.

\section{INTRODUÇÃO}

Segundo dados do último censo agropecuário do Instituto Brasileiro de Geografia e Estatística (IBGE), no Brasil, o efe- 
tivo de caprinos foi de 9,16 milhões de cabeças. A região Sul apresentou o segundo maior rebanho, 335.720 cabeças, com o Rio Grande do Sul (RS) totalizando 101.420 cabeças. A região Nordeste deteve o maior número de cabeças caprinas, totalizando 8,3 milhões (Brasil 2009). A criação de caprinos abrange a caprinocultura de corte e de leite e esses dois ramos estão em crescimento no mercado financeiro brasileiro (Medeiros et al. 2009).

Não há estudos retrospectivos abrangentes das causas de morte de caprinos no RS, não havendo também como estimar as perdas econômicas relacionadas. Vários estudos têm sido realizados sobre doenças ou grupos de doenças de caprinos no Brasil (Bandarra et al. 2011, Loretti et al. 2003, Rodrigues et al. 2007, Riet-Correa et al. 2008, Medeiros et al. 2012). No entanto, há uma carência de estudos abrangentes de todas as doenças diagnosticadas na população caprina com sua respectiva prevalência em determinado estado ou região.

Os objetivos deste estudo foram determinar a prevalência e as características epidemiológicas e clínico-patológicas das doenças que acometem caprinos na área de abrangência do Laboratório de Patologia Veterinária da Universidade Federal de Santa Maria (LPV-UFSM), localizado na região Central do RS. Para isso foram consultados os arquivos de necropsias do LPV-UFSM e agrupadas as informações referentes aos casos de doença de caprinos no período de 1964 a 2011.

\section{MATERIAL E MÉTODOS}

Os laudos de necropsias de caprinos realizadas entre janeiro de 1964 e dezembro de 2011 no LPV-UFSM foram revisados. Casos experimentais foram excluídos do estudo. Desses laudos foram coletados os dados epidemiológicos e os achados clínico-patológicos dos caprinos necropsiados. Os diagnósticos foram classificados em conclusivos e inconclusivos. Os casos com diagnóstico conclusivo foram agrupados, de acordo com a etiologia, em: 1) intoxicações e toxi-infecções, 2) doenças metabólicas e nutricionais, 3) doenças infecciosas e parasitárias, e 4) alterações do desenvolvimento. Outras condições ou lesões que não se enquadravam em nenhum dos grupos de doenças acima foram classificadas como "outras alterações".

\section{RESULTADOS}

No período estudado (48 anos), foram realizadas 119 necropsias de caprinos. Cinco casos eram provenientes de experimentos e foram excluídos do estudo, resultando em 114 necropsias. Em 30 (26,31\%) casos não havia informação sobre a raça. Dos 84 caprinos que tiveram esse parâmetro informado nos protocolos de necropsia, havia 49 (58,33\%) sem raça definida e 35 (41,66\%) de raças puras, que incluíam: Boer (18/84 [21,42\%]), Sannen (13/84 [15,47\%]), Parda Alpina (2/84 [2,38\%]), Mambrina (1/84 [1,19\%]) e Anglo Nubiana (1/84 [1,19\%]). Do total de caprinos que tiveram o sexo informado nos protocolos de necropsia $(n=111), 65$ (58,56\%) eram fêmeas e $46(41,44 \%)$ eram machos. A idade variou entre neonatos e 8 anos de idade.

Do total de necropsias de caprinos, 95 (83,33\%) tinham diagnóstico conclusivo e 19 (19,66\%) tinham diagnóstico inconclusivo. Dos 95 casos conclusivos, 46 (48,42\%) eram de doenças infecciosas e parasitárias; $26(27,36 \%)$ eram de doenças metabólicas e nutricionais; $10(10,52 \%)$ eram de intoxicações e toxi-infecções; e um $(1,05 \%)$ era de alterações do desenvolvimento. Outras alterações foram diagnosticadas em 12 caprinos (12,63\%). A distribuição dos casos em cada um dos grupos encontra-se no Quadro 1.

As doenças infecciosas e parasitárias foram as mais prevalentes (40,35\% do total de diagnósticos conclusivos). Nesse grupo, hemoncose $(n=21)$, eimeriose $(n=9)$ e listeriose $(n=9)$ predominaram. Dentre as demais condições nesse grupo $(\mathrm{n}=7)$, três delas afetaram o sistema respiratório, incluindo pneumonias/broncopneumonias de provável origem bacteriana e um caso de pneumonia verminótica por Muellerius capillaris.

Quadro 1. Doenças de caprinos diagnosticadas no Laboratório de Patologia Veterinária, Universidade Federal de Santa Maria, entre 1964 e 2011

\begin{tabular}{|c|c|c|c|}
\hline Doenças infecciosas e parasitárias & $\mathrm{N}$ & $\begin{array}{c}\% \text { da } \\
\text { categoria }\end{array}$ & $\begin{array}{l}\% \text { do } \\
\text { total }\end{array}$ \\
\hline Hemoncose & 21 & 45,65 & 18,42 \\
\hline Eimeriose & 9 & 19,56 & 7,89 \\
\hline Listeriose & 9 & 19,56 & 7,89 \\
\hline Hepatite necrótica & 1 & 2,17 & 0,87 \\
\hline Broncopneumonia supurativa & 1 & 2,17 & 0,87 \\
\hline Pleuropneumonia fibrinosa & 1 & 2,17 & 0,87 \\
\hline Pododermatite & 1 & 2,17 & 0,87 \\
\hline Pneumonia por Muellerius capillaris & 1 & 2,17 & 0,87 \\
\hline Aborto por Neospora sp. & 1 & 2,17 & 0,87 \\
\hline Abcesso cerebral & 1 & 2,17 & 0,87 \\
\hline TOTAL & 46 & 100 & 40,35 \\
\hline Doenças metabólicas e nutricionais & $\mathrm{N}$ & $\begin{array}{c}\% \mathrm{da} \\
\text { categoria }\end{array}$ & $\begin{array}{l}\% \text { do } \\
\text { total }\end{array}$ \\
\hline Urolitíase & 5 & 19,23 & 4,38 \\
\hline Osteoporose & 5 & 19,23 & 4,38 \\
\hline Toxemia da prenhez & 4 & 15,38 & 3,50 \\
\hline Desnutrição & 4 & 15,38 & 3,50 \\
\hline Doença dos músculos brancos & 3 & 11,53 & 2,63 \\
\hline Acidose ruminal & 2 & 7,69 & 1,75 \\
\hline Osteodistrofia fibrosa (nutricional) & 1 & 3,84 & 0,87 \\
\hline Deficiência de cobre & 1 & 3,84 & 0,87 \\
\hline Polioencefalomalácia & 1 & 3,84 & 0,87 \\
\hline TOTAL & 26 & 100 & 22,80 \\
\hline Intoxicações e toxi-infecções & $\mathrm{N}$ & $\begin{array}{c}\% \text { da } \\
\text { categoria }\end{array}$ & $\begin{array}{l}\% \text { do } \\
\text { total }\end{array}$ \\
\hline Intoxicação por closantel & 7 & 70 & 6,14 \\
\hline Intoxicação por Prunus sp. & 1 & 10 & 0,87 \\
\hline Necrose hepática centrolobular & 1 & 10 & 0,87 \\
\hline Tétano & 1 & 10 & 0,87 \\
\hline TOTAL & 10 & 100 & 8,77 \\
\hline Alterações do desenvolvimento & $\mathrm{N}$ & $\begin{array}{c}\% \text { da } \\
\text { categoria }\end{array}$ & $\begin{array}{l}\% \text { do } \\
\text { total }\end{array}$ \\
\hline Defeito cardíaco de septo atrial & 1 & 100 & 0,87 \\
\hline Outras alterações & $\mathrm{N}$ & $\begin{array}{c}\% \text { da } \\
\text { categoria }\end{array}$ & $\begin{array}{l}\% \text { do } \\
\text { total }\end{array}$ \\
\hline Úlcera de abomaso com peritonite & 3 & 25 & 2,63 \\
\hline Intussuscepção intestinal & 2 & 16,66 & 1,75 \\
\hline Espongiose da substância brancaencefálica & 2 & 16,66 & 1,75 \\
\hline Timpanismo & 1 & 8,33 & 0,87 \\
\hline Vólvulo intestinal & 1 & 8,33 & 0,87 \\
\hline Úlcera duodenal com peritonite & 1 & 8,33 & 0,87 \\
\hline Peritonite & 1 & 8,33 & 0,87 \\
\hline Choque séptico & 1 & 8,33 & 0,87 \\
\hline TOTAL & 12 & 100 & 10,52 \\
\hline TOTAL GERAL & 114 & - & 100,00 \\
\hline
\end{tabular}


A condição mais prevalente no grupo $(45,65 \%)$ e sobre o total de diagnósticos $(18,42 \%)$ foi a hemoncose. A idade dos animais afetados foi de seis meses a cinco anos; em 10 casos os caprinos tinham dois anos. Os principais sinais clínicos apresentados foram: emagrecimento, apatia, edema subcutâneo da região submandibular, anemia e diarreia. Nos históricos de 10 casos havia informações de que outros caprinos das mesmas propriedades apresentavam os mesmos sinais clínicos. Os achados de necropsia mais frequentes foram palidez das mucosas (anemia), sangue de aspecto aquoso, ascite, hidropericárdio, edema pulmonar, palidez das vísceras da cavidade abdominal, atrofia serosa da gordura e presença de grande quantidade de parasitas (morfologicamente compatíveis com Haemonchus sp.) no abomaso. Microscopicamente observou-se degeneração e/ou necrose hepatocelular centrolobular na maioria dos casos.

A eimeriose, igualando-se à listeriose, foi a segunda doença mais prevalente no grupo $(19,56 \%)$ e sobre o total $(7,89 \%)$. Em cinco casos os caprinos eram provenientes de criações intensivas e com alta densidade populacional. Em todos os casos de eimeriose foram observados sinais clínicos de doença crônica, caracterizada por emagrecimento e diarreia. Em cinco casos a diarreia era vermelho-escura e em quatro casos foi observada palidez das mucosas (anemia). A idade dos animais acometidos variou de dois a seis meses. Em dois casos, a doença se manifestou em forma de surtos, pois outros caprinos das mesmas propriedades foram afetados. Na necropsia, os achados mais frequentes foram intestino com nódulos brancos levemente elevados na mucosa e com conteúdo mucoso ou líquido e ocasionalmente avermelhado, palidez das mucosas e vísceras e edema subcutâneo na região submandibular. Menos comumente foram observados atrofia serosa da gordura e edema pulmonar. Microscopicamente, nos intestinos delgado e grosso, foram observadas áreas multifocais do epitélio com acentuada quantidade de parasitas protozoários em diferentes estágios de desenvolvimento, incluindo oocistos, e morfologicamente compatíveis com Eimeria sp. Na lâmina própria, havia acentuado infiltrado inflamatório composto por linfócitos, plasmócitos e macrófagos.

Todos os casos de listeriose constituíram-se da forma nervosa da doença. A idade dos animais afetados variou de três meses a quatro anos. Todos os casos ocorreram na primavera ou verão. Em quatro dos nove casos, foi fornecida silagem para os caprinos. Em seis casos a doença se manifestou em forma de surtos, com envolvimentos que variaram de três a 12 caprinos por propriedade, com sinais clínicos semelhantes. Os sinais clínicos observados com maior frequência foram: desvio lateral da cabeça, andar em círculos, decúbito, cegueira e nistagmo. Quando informado, o curso clínico variou de 2 a 10 dias, com exceção de um caso onde foi de 30 dias. Não foram observadas lesões macroscópicas no encéfalo em nenhum dos casos. Microscopicamente observou-se meningoencefalite não-supurativa (predominantemente no tronco encefálico), com microabcessos e infiltração de células gitter (malacia).

Dentre as doenças metabólicas e nutricionais, o segundo grupo mais prevalente $(22,8 \%$ do total de diagnósticos conclusivos), predominaram a urolitíase $(\mathrm{n}=5)$, a osteoporose $(\mathrm{n}=5)$, a toxemia da prenhez $(\mathrm{n}=4)$, a desnutrição $(n=4)$ e a doença dos músculos brancos $(n=3)$.

A idade dos caprinos com urolitíase $(19,23 \%$ no grupo e $4,38 \%$ do total) variou de 40 dias a quatro anos, sendo três deles com cinco meses de idade. Todos os cinco eram machos; entretanto, nenhum era castrado. Em todos os casos era fornecida ração composta principalmente por grãos para os caprinos. Em um dos casos, outros sete caprinos na propriedade estavam com os mesmos sinais clínicos. 0 curso clínico variou de dois a quatro dias. Os principais sinais clínicos apresentados foram dor abdominal aguda, anorexia, dificuldade para andar, marcha rígida, edema de prepúcio, disúria, anemia em três casos (não associada a parasitismo) e anúria por três dias em um dos casos. Os achados de necropsia mais frequentes foram uretrite hemorrágica, uretrite fibrinopurulenta, obstrução uretral, necrose do apêndice vermiforme, cistite fibrinonecro-hemorrágica (com coágulos de sangue), peritonite e, em um dos casos, hidronefrose bilateral e, em outro, ruptura de bexiga com uroperitônio.

Osteoporose $(19,23 \%$ no grupo e $4,38 \%$ do total) foi diagnosticada em cinco cabras em uma propriedade de baixa condição sanitária, com alta densidade populacional e com cabras de diferentes idades dividindo as mesmas instalações. A idade das cabras afetadas variou de seis meses a seis anos e os principais sinais clínicos foram claudicação, relutância em levantar ou andar e acentuada desnutrição. Na necropsia, os ossos longos e vértebras foram examinados na sua superfície de corte e observou-se depleção de osso esponjoso metafisário e epifisário, tornando o osso mais poroso que o normal, diminuição acentuada da espessura do córtex ósseo diafisário principalmente e acentuada atrofia serosa da gordura da medula óssea. Parasitismo por $H$. contortus no abomaso também foi observado. Microscopicamente, havia marcada osteoporose, caracterizada por diminuição do número e espessura das trabéculas ósseas nas epífises e metáfises dos ossos longos e nos corpos vertebrais.

Os casos de toxemia da prenhez $(15,38 \%$ no grupo e $3,50 \%$ do total) afetaram cabras de 1-3 anos de idade. Em três casos a gestação era gemelar e em um caso era trigemelar. Todos os casos da doença aconteceram no final da gestação e em dois casos as fêmeas estavam em bom estado nutricional. Os sinais clínicos apresentados foram, prostração, anorexia, dificuldade respiratória, convulsão e, em um caso, a cabra estava em coma quando foi examinada. 0 curso clínico não foi informado em nenhum dos casos. Na necropsia o fígado apresentava-se amarelado, friável, e as lesões histológicas caracterizavam-se por marcada degeneração gordurosa dos hepatócitos.

Os casos de desnutrição (15,38\% no grupo e 3,50\% do total) afetaram caprinos de 1-6 meses de idade. Em dois casos outros caprinos das propriedades morreram com os mesmos sinais clínicos. Os principais sinais apresentados foram diarreia, anorexia e fraqueza. Na necropsia, os caprinos apresentavam-se em mau estado nutricional, ausência de tecido adiposo visceral e atrofia das massas musculares. 
A doença dos músculos brancos $(11,53 \%$ no grupo e $2,63 \%$ do total) acometeu caprinos com idade entre 14 dias e um mês. Em todos os casos adoeceram e morreram grande número de animais com quadro clínico semelhante na mesma propriedade, sendo que em dois casos os animais tinham a mesma procedência. Os sinais clínicos observados foram depressão, fraqueza, quedas frequentes e incoordenação. Na necropsia, os músculos das grandes massas musculares dos membros pélvicos estavam pálidos em dois casos; em um caso não se observaram alterações macroscópicas. Microscopicamente foi observada necrose muscular caracterizada por miofibras fragmentadas, com um aspecto flocular, infiltradas por macrófagos (necrose flocular) e algumas miofibras com citoplasma acentuadamente eosinofílico, com perda das estriações e deposição de material basofílico granular (mineralização).

0 terceiro grupo, em ordem decrescente de prevalência, foi o das intoxicações e toxi-infecções $(8,77 \%$ do total de diagnósticos conclusivos), onde a intoxicação por closantel $(n=7)$ foi a mais prevalente. Os sete casos de intoxicação pelo anti-parasitário closantel foram provenientes de um surto onde os caprinos receberam superdosagem do medicamento. Os caprinos tinham entre sete e oito meses de idade. 0 curso clínico da doença foi de um a dois dias e os caprinos apresentaram sintomatologia nervosa, caracterizada por cegueira, opistótono, nistagmo, tremores, midríase, decúbito lateral e morte. As demais intoxicações e toxi-infecções, incluiram intoxicação por Prunus sp., necrose hepática centrolobular (padrão tóxico, porém a causa específica não foi determinada) e tétano, e tiveram uma ocorrência cada.

Apenas um caprino apresentou alteração do desenvolvimento $(0,87 \%$ do total de diagnósticos conclusivos) constituída por defeito cardíaco no septo atrial. Como "outras alterações" (10,52\% do total de diagnósticos conclusivos), observou-se um grupo heterogêneo de condições ou lesões que afetaram predominantemente o sistema digestório (9/12).

\section{DISCUSSÃO}

Este levantamento permitiu que se estabelecessem as características epidemiológicas, incluindo as raças presentes no rebanho caprino da área de abrangência do LPV-UFSM, bem como a prevalência dos principais grupos de etiologias e doenças específicas que acometeram esse rebanho.

As doenças infecciosas e parasitárias foram as diagnosticadas com maior frequência. A hemoncose e a eimeriose, doenças parasitárias do trato gastrointestinal, foram as doenças diagnosticadas com maior frequência, demonstrando sua grande importância, pois causam mortalidade e, principalmente, comprometem o processo produtivo, com redução do ganho de peso, na produção de leite, na qualidade da carcaça, em especial nas situações em que a nutrição é deficiente (Medeiros et al. 2009). A hemoncose foi também a doença mais prevalente em um estudo retrospectivo feito na espécie ovina no LPV-UFSM (Rissi et al. 2010b). Esta parasitose ocorre em locais de verões chuvosos, particularmente em regiões tropicais e subtropicais (Cavalcante et al. 2010). 0 diagnóstico de hemoncose nos casos aqui es- tudados foi realizado pelos achados clínico-patológicos característicos (Brown et al. 2007), o qual é facilitado quando se observa grande número de exemplares do nematódeo (Haemonchus contortus) no abomaso, permitindo que sejam empregadas medidas eficazes de controle e tratamento no rebanho. Entretanto, ausência dos parasitas no abomaso pode ser devido à autólise ou tratamento recente com anti-parasitários (Radostits et al. 2007a). Diagnósticos diferenciais para anemia em caprinos incluem: leptospirose, intoxicação por cobre, coccidiose, fasciolose, deficiência de cobalto, deficiência de fósforo e doenças crônicas (paratuberculose) (Navarre \& Pugh 2002).

Em cinco dos nove casos de eimeriose foram observados sinais clínicos clássicos da doença, já descritos por outros autores (Vieira \& Berne 2007). As principais espécies do gênero Eimeria que causam doença em caprinos são: $E$. arloingi, E. christenseni e E. ninakohlyakimovae. Caprinos, especialmente até oito meses de idade, são os mais susceptíveis à infecção e, geralmente, apresentam os sinais mais acentuados da protozoose (Brown et al. 2007). Todos os caprinos com eimeriose deste estudo eram jovens (2-6 meses). Essa protozoose é mais frequente em criações intensivas e com alta densidade populacional (Vieira \& Berne 2007), como descrita no histórico clínico de cinco dos nove casos aqui estudados. Nódulos brancos levemente elevados na mucosa do intestino são característicos da doença (Brown et al. 2007). Os sinais clínicos observados nos casos de hemoncose e eimeriose foram semelhantes, entretanto, diarreia escura e os achados macroscópicos e microscópicos no intestino só foram observados nos casos de eimeriose. Rápidas medidas de controle e tratamento no restante dos caprinos reduz as perdas econômicas decorrentes da doença.

Nos nove casos de listeriose a epidemiologia, os sinais clínicos e as lesões histopatológicas observadas eram característicos (Barros et al. 2006, Low \& Donachie 1997, Rissi et al. 2006). Em seis casos a doença se manifestou em forma de surtos, com envolvimento de vários caprinos por propriedade. Portanto, a listeriose é uma doença de grande importância para caprinos na região Central do Rio Grande do Sul. Dados epidemiológicos e clínico-patológicos de três dos nove casos aqui descritos foram previamente publicados (Rissi et al. 2006). Em animais, Listeria monocytogenes, pode induzir três formas de doença: septicemia, com formação de abscessos em vários órgãos, abortos ou doença neurológica caracterizada por meningoencefalite e formação de microabscessos no tronco encefálico (George 2006). A época de ocorrência da doença foi na primavera e verão, semelhante aos casos de listeriose em ovinos no RS (Rissi et al. 2010a). Somente em quatro dos nove casos, foi fornecida silagem para os caprinos. Sabe-se que a doença neurológica é mais frequente no inverno e início da primavera, e tem sido associada à alimentação com silagem de má qualidade ( $\mathrm{pH}$ acima de 5,5), que favorece o crescimento da bactéria (Low \& Donachie 1997). Entretanto, surtos de listeriose em ovinos no sul da Austrália (Reuter et al. 1989), onde os casos ocorreram entre janeiro e abril, raramente estavam associados à alimentação com silagem. 0 diagnóstico nos casos de listeriose foi realizado com base 
na epidemiologia e lesões microscópicas características da doença; em alguns desses casos foi realizado o teste de imuno-histoquímica com resultados positivos para Listeria sp. (Rissi et al. 2006).

Dentre as doenças metabólicas e nutricionais, as mais prevalentes foram a urolitíase, a osteoporose e a toxemia da prenhez. A prevalência da urolitíase é mais alta em ovinos e caprinos machos castrados em idade precoce, por terem menor desenvolvimento da uretra, que é de menor calibre que a uretra de animais não castrados, e em animais alimentados com dietas ricas em grãos com proporção de Ca:P de aproximadamente 1:1 ou menor (Riet-Correa 2007). Neste estudo, todos os caprinos afetados eram machos; entretanto, nenhum era castrado, mas em todos os casos era fornecida ração composta principalmente por grãos para os caprinos. Portanto, urolitíase pode ser um problema de manejo, acometendo vários animais numa propriedade, como visto neste estudo. Os sinais clínicos apresentados foram característicos da condição (Riet-Correa et al. 2008). No entanto, anemia não é um sinal clínico comumente descrito em casos de urolitíase. Nos casos deste estudo é provável que a anemia tenha sido consequência de hematúria grave, decorrente de cistite hemorrágica acentuada com formação de coágulos que recobriam a mucosa vesical. Somente em dois caprinos foram encontrados os urólitos, em ambos na uretra peniana. Em dois casos observou-se ausência do apêndice vermiforme, o que sugere necrose causada por obstrução uretral cranialmente (ou secundário a procedimento cirúrgico terapêutico não informado no histórico clínico dos animais). Nem sempre os urólitos são vistos na necropsia nos casos de urolitíase, pois podem ser liberados espontaneamente antes do animal morrer ou por sondagens uretrais (Loretti et al. 2003). Em ruminantes a flexura sigmoide, a curvatura isquiática, e o apêndice vermiforme constituem os locais mais comuns para os urólitos se alojarem (Radostits et al. 2007b).

Quanto à ocorrência de osteoporose, um estudo detalhado dos dados epidemiológicos, clínicos e patológicos dos casos deste levantamento serão alvo de outra publicação.

Toxemia da prenhez é frequentemente observada em ovinos e caprinos com prenhez múltipla e no último mês da gestação, como observado nos casos deste estudo. No entanto, essa condição pode ocorrer na gestação de um único feto muito desenvolvido (Schild 2007), ou em animais que pariram recentemente. Os sinais clínicos em cabras e ovelhas são geralmente da forma nervosa da doença; inicialmente os animais separam-se do restante do rebanho e aparentam cegueira; se forem forçados a andar, batem-se em obstáculos ou pressionam a cabeça contra os mesmos (Mobini et al. 2002). Nenhum desses sinais foram observados nos casos deste estudo. Entretanto, convulsão foi observada em dois casos, o que é geralmente descrito no estágio final da doença (Mobini et al. 2002). Os sinais nervosos são decorrentes de hipoglicemia, causada pela maior demanda de glicose durante a gestação gemelar (Schild 2007). Microscopicamente, no encéfalo, não foram observadas lesões de hipoglicemia nos casos deste estudo. 0 diagnóstico de toxemia da prenhez foi feito com base na avaliação dos aspectos epidemiológicos, sinais clínicos, pelos achados de necropsia e histológicos. Os diagnósticos diferenciais incluem hipocalcemia ou hipomagnesemia, e doenças que cursam com sinais neurológicos como polioencefalomalacia, enterotoxemia, raiva e listeriose (Schild 2007).

Dentre as demais doenças metabólicas e nutricionais, vale ressaltar a ocorrência da doença dos músculos brancos, pouco frequente nessa espécie, porém já diagnosticada no Rio Grande do Sul (RS) (Loretti et al. 2001) e sendo a causa de morte mais importante em caprinos jovens em estudos realizados na Nova Zelândia (Buddle et al. 1988) e no México (Ramíres-Bribiesca et al. 2001). A doença, também conhecida como miopatia nutricional, afeta mais comumente bezerros, cordeiros, suínos e potros, porém cabritos também são suscetíveis à doença. As deficiências nutricionais são principalmente de selênio (Se) e vitamina E. A doença geralmente afeta animais jovens em desenvolvimento, mantidos em pastos cujos solos são pobres em Se e vitamina $\mathrm{E}$ ou que recebem rações deficientes nesses elementos e comumente ocorre em forma de surtos (Van Vleet \& Valentine 2007). Nos caprinos deste estudo outros caprinos da mesma propriedade foram afetados ressaltando a importância no diagnóstico da doença em caprinos no RS. Outras doenças consideradas como diagnóstico diferencial são as miopatias tóxicas como as intoxicações por Senna occidentalis e antibióticos ionóforos, no entanto, estas foram descartadas, pois no histórico dos caprinos não havia informação de administração de antibióticos ionóforos na ração nem a presença da planta na propriedade ou na ração.

Quanto às intoxicações e toxi-infecções diagnosticadas nos caprinos deste estudo, os dados referentes à epidemiologia e os achados clínico-patológicos do surto de intoxicação por closantel foram previamente publicados (Ecco et al. 2006). Experimento posterior a esse surto detalhou as alterações oftálmicas associadas à intoxicação experimental por closantel em caprinos (Ecco et al. 2008). Outro surto com aspectos epidemiológicos e clínico-patológicos semelhantes foi descrito em Santa Catarina (Furlan et al. 2009).

\section{CONCLUSÕES}

Através deste estudo retrospectivo pode-se concluir que:

- As doenças infecciosas e parasitárias foram as mais prevalentes nos caprinos examinados, seguidas em ordem decrescente de prevalência, pelas doenças metabólicas e nutricionais, intoxicações e toxi-infecções e alterações do desenvolvimento;

- Outras alterações de diferentes naturezas e etiologias afetaram cerca de $10 \%$ dos caprinos examinados;

- A hemoncose é a principal causa de morte de caprinos na área de abrangência do LPV-UFSM;

- Eimeriose e listeriose também são causas importantes de morte de caprinos nessa área;

- Principalmente as doenças infecciosas e parasitárias e as metabólicas e nutricionais ocorreram muitas vezes na forma de surtos, acarretando maiores perdas econômicas aos criadores de caprinos da região.

Agradecimentos.- F.B. Rosa é bolsista do Conselho Nacional de Desenvolvimento Científico e Tecnológico (CNPq). G.J.N. de Galiza é bolsista da Coordenação de Aperfeiçoamento de Pessoal de Nível Superior (CAPES). R.A. 
Caprioli e T.M. Silva são bolsistas PIBIC/CNPq. G.D. Kommers é bolsista de produtividade em pesquisa (PQ-2) do CNPq.

\section{REFERÊNCIAS}

Bandarra P.M., Pavarini S.P., Santos A.S., Antoniassi N.A.B., Cruz C.E.F. \& Driemeier D. 2011. Osteodistrofia fibrosa nutricional em caprinos. Pesq. Vet. Bras. 31(10):875-878.

Barros C.S.L., Driemeier D., Dutra I.S. \& Lemos R.A.A. 2006. Listeriose, p.77-80. In: Ibid. (Eds), Doenças do Sistema Nervoso de Bovinos no Brasil. Vallée, São Paulo, SP.

Brasil 2009. Instituto Brasileiro de Geografia e Estatística (IBGE). Disponível em <http://www.ibge.gov.br> Acessado em 4 abr. 2012.

Brown C.C., Baker D.C. \& Barker I.K. 2007. Alimentary system, p.1-296. In: Maxie M.G. (Ed.), Jubb, Kennedy and Palmer's Pathology of Domestic Animals. Vol.2. $5^{\text {th }}$ ed. Elsevier, Philadelphia.

Buddle B.M., Herceg M., Ralston M.J., Pulford H.D., Millar K.R. \& Elliott D.C. 1988. A goat mortality study in the southern North Island. N.Z. Vet. J. 36(4):167-170.

Cavalcante A.C.R., Vieira L.S., Chagas A.C.S. \& Molento M.B. 2010. Doenças Parasitárias de Caprinos e Ovinos: epidemiologia e controle. Embrapa, Brasília, DF. 604p.

Ecco R., Barros C.S.L. \& Graça D.L. 2008. Alterações oftálmicas associadas à intoxicação experimental por closantel em caprinos. Arq. Bras. Med. Vet. Zootec. 60(1): 42-50.

Ecco R., Barros C.S.L., Graça D.L. \& Gava A. 2006. Closantel toxicosis in kid goats. Vet. Rec. 159(17):564-566.

Furlan F.H., Lucioli J., Borelli V., Fonteque J.H., Stolf L., Traverso S.D. \& Gava A. 2009. Intoxicação por closantel em ovinos e caprinos no Estado de Santa Catarina. Pesq. Vet. Bras. 29(1):89-93.

George L.W. 2006. Listeriose, p.946-949. In: Smith B.P. (Ed.), Medicina Interna de Grandes Animais. 3a ed. Manole, Barueri, SP.

Loretti A.P., Driemeier D., Traverso S.D. \& Seitz A.L. 2001. Miopatia nutricional em caprinos no Rio Grande do Sul. Anais X Encontro Nacional de Patologia Veterinária, Pirassununga, SP, p.36. (Resumo)

Loretti A.P., Oliveira L.O., Cruz C.E.F. \& Driemeier D. 2003. Clinical and pathological study of obstructive urolithiasis in feedlot cattle in Southern Brazil. Pesq. Vet. Bras. 23(2):61-64.

Low J.C. \& Donachie W. 1997. A review of Listeria monocytogenes and listeriosis. Vet. J. 153(1):9-29.

Medeiros J.M.A., Garino Jr F., Almeida A.P., Lucena E.A. \& Riet-Correa F. 2012. Paratuberculose em caprinos e ovinos no Estado da Paraíba. Pesq. Vet. Bras. 32(2):111-115.

Medeiros L.P., Girão R.N., Girão E.S. \& Leal J.A. 2009. Caprinos, o produtor pergunta, a Embrapa responde. Embrapa, Brasília, DF. 170p.

Mobini S., Heath A.M. \& Pugh D.G. 2002. Theriogenology of sheep and goats, p.129-187. In: Pugh D.G. (Ed.), Sheep and Goat Medicine. Elsevier, Philadelphia.

Navarre C.B. \& Pugh D.G. 2002. Diseases of the gastrointestinal system, p.69-105. In: Pugh D.G. (Ed.), Sheep and Goat Medicine. Elsevier, Philadelphia.

Radostits O.M., Gay C.C., Hinchcliff K.W. \& Constable P.D. 2007a. Hemoncosis in ruminants, p.1548-1552. In: Ibid. (Eds), Veterinary Medicine: a textbook of the diseases of cattle, horses, sheep, pigs, and goats. $10^{\text {th }}$ ed. W.B. Saunders, Philadelphia.

Radostits O.M., Gay C.C., Hinchcliff K.W. \& Constable P.D. 2007b. Urolithiasis in ruminants, p.565-570. In: Ibid. (Eds), Veterinary Medicine: a textbook of the diseases of cattle, horses, sheep, pigs, and goats. $10^{\text {th }} \mathrm{ed}$. W.B. Saunders, Philadelphia.

Ramíres-Bribiesca J.E., Tórtora J.L., Hernández L.M. \& Huerta M. 2001. Main causes of mortalities in dairy goat kids from the Mexican plateau. Small Rumin. Res. 41:77-80.

Reuter R., Bowden M. \& Palmer M. 1989. Ovine listeriosis in south coastal Western Australia. Aust. Vet. J. 66(7):223-224.

Riet-Correa F. 2007. Urolitíase em ruminantes, p.677-684. In: Riet-Correa F., Schild A.L., Lemos R.A.A. \& Borges J.R.J. (Eds), Doenças de Ruminantes e Eqüídeos. Vol.2. $3^{a}$ ed. Pallotti, Santa Maria, RS.

Riet-Correa F., Simões S.V.D. \& Vasconcelos J.S. 2008. Urolitíase em caprinos e ovinos. Pesq. Vet. Bras. 28(6):319-322.

Rissi D.R., Kommers G.D., Marcolongo-Pereira C., Schild A.L. \& Barros C.S.L. 2010a. Meningoencefalite por Listeria monocytogenes em ovinos. Pesq. Vet. Bras. 30(1):51-56.

Rissi D.R., Pierezan F., Filho J.C.O., Fighera R.A., Irigoyen L.F., Kommers G.D. \& Barros C.S.L. 2010b. Doença de ovinos da região Central do Rio Grande do Sul: 361 casos. Pesq. Vet. Bras. 30(1):21-28.

Rissi D.R., Rech R.R., Barros R.R., Kommers G.D., Langohr I.M., Pierezan F. \& Barros C.S.L. 2006. Forma nervosa de listeriose em caprinos. Pesq. Vet. Bras. 26(1):14-20.

Rodrigues A.B., Athayde A.C.R., Rodrigues O.G., Silva W.W. \& Faria E.B. 2007. Sensibilidade dos nematoides gastrintestinais de caprinos a antihelmínticos na mesorregião do Sertão Paraibano. Pesq. Vet. Bras. 27(4): 162-166.

Schild A.L. 2007. Cetose, p.281-286. In: Riet-Correa F., Schild A.L., Lemos R.A.A. \& Borges J.R.J. (Eds), Doenças de Ruminantes e Eqüídeos. Vol.2. $3^{\text {ạ }}$ ed. Pallotti, Santa Maria, RS.

Van Vleet J.F. \& Valentine B.A. 2007. Muscle and tendon, p.185-280. In: Maxie M.G. (Ed.), Jubb, Kennedy and Palmer's Pathology of Domestic Animals. Vol.1. $5^{\text {th }}$ ed. Elsevier, Philadelphia.

Vieira L.S. \& Berne M.E.A. 2007. Eimeriose de caprinos e ovinos, p.666673. In: Riet-Correa F., Schild A.L., Lemos R.A.A. \& Borges J.R.J. (Eds), Doenças de Ruminantes e Eqüídeos. Vol.1. 3a ed. Pallotti, Santa Maria, RS. 